\title{
Acute Hemiplegia Associated With Cat-Scratch Disease
}

\author{
Rocha J.L., Pellegrino L.N., Riella L.V., Martins L.T.
}

Department of Infectious Disease of the Nossa Senhora das Graças Hospital, Curitiba, PR, Brazil

\begin{abstract}
Cat scratch disease (CSD) is an infectious illness caused by a Gram-negative rod named Bartonella henselae. Typical CSD is characterized by a small skin lesion at the site of a scratch or a bite, followed by regional lymphadenopathy, one to two weeks later. Atypical forms may present as ocular manifestations, neurological manifestations, hepatosplenic involvement and vertebral osteomyelitis. Among neurological complications, encephalopathy is by far the most common. Other neurological manifestations are very rare. We report a case of an 11-year-old boy, with a posterior cervical lymphadenopathy and fever. Cat scratch disease was diagnosed and treated after a positive "Whartin-Starry" stain on lymph node biopsy. Two weeks after treatment, the patient was readmitted presenting an acute episode of left hemiplegia. A brain MRI demonstrated a right subcortical fronto-parietal lesion with no contrast enhancement. Complete recovery was observed after corticosteroid treatment.

Key Words: Cat-scratch disease, acute hemiplegia, corticosteroid treatment.
\end{abstract}

Cat scratch disease (CSD), also called cat scratch fever and benign lymphoreticulosis, is an infectious illness associated with cat scratches, bites or exposure to cat saliva, causing self-limited regional lymphadenopathy.

The causative organism of CSD, in most cases, is a Gram-negative rod - Bartonella henselae, formerly known as Rochalimaea henselae [1]. There have also been a few rare reports of CSD due to Afipia felis and Bartonella clarridgeiae.

Typical CSD initially develops as a small skin lesion, resembling an insect bite, at the site of a scratch or bite. A round, red-brown, nontender papule develops in the scratch line after 3 to 10 days. Less commonly, the primary inoculation lesion can be pustular or nodular. It may vary in size from one to several millimeters and may persist for about one to three weeks [1]. One to

Received on 15 February 2004; revised 10 May 2004.

Address for correspondence: Dr. Jaime Luis Rocha-152, Urbano Lopes St. apt. 1501 80050-520 Curitiba - PR, Brazil - Phone: 55-41-9965-3355 - email: jaimeluis@ b.com.br

The Brazilian Journal of Infectious Diseases 2004;8(3):263-266 (C) 2004 by The Brazilian Journal of Infectious Diseases and Contexto Publishing. All rights reserved. two weeks later, one or more regional lymph nodes that drain the area gradually enlarge. Regional lymphadenopathy is the hallmark of CSD.

Atypical forms of CSD are characterized by ocular or neurological manifestations, hepatosplenic involvement, vertebral osteomyelitis and other atypical manifestations [1-3]. Although the primary diagnosis of CSD is clinical, it can be diagnosed reliably with serologic testing and histopathology.

\section{Case Report}

An 11-year-old boy was admitted to the hospital because of a posterior cervical lymphadenopathy and fever. The patient was asymptomatic until one week earlier, when a firm and tender left posterior cervical lymph node was noted. Symptomatic treatment was ineffective. One week before admission, daily fever was noticed. After progressive worsening, the patient was admitted to the hospital.

The patient had no past medical history of concern, except for an amygdalectomy at the age of five. He had no recent history of pharyngitis and was not taking 
any medicine. He had not traveled recently. He denied the ingestion of undercooked meat. He had been exposed to cats, but no bites or scratches were reported.

Axillary body temperature was over $39^{\circ} \mathrm{C}$ in several measurements on the first two days There was a single lymph node in the left posterior cervical chain, $5 \mathrm{~cm}$ in diameter, not fluctuant or accompanied by overlying erythema. On examination no skin lesions were found. Neurological exam was normal.

Complete blood count and biochemical panel were normal. Erythrocyte sedimentation rate was $91 \mathrm{~mm} / \mathrm{h}$; C-reactive protein was 46mg/dL. Serology was IgG positive only for: toxoplasmosis, CMV and EBV; HIV was negative.

The cerebrospinal fluid analysis showed: eight leukocytes, with a predominance of monomorphonuclear cells, $32.3 \mathrm{mg} / \mathrm{dL}$ proteins, and $58.5 \mathrm{mg} / \mathrm{dL}$ glucose. Urine exam was normal. Hemocultures yielded no microorganisms. Chest x-ray was normal. Abdominal ultra-sound showed a light hepatoesplenomegaly and adenomegaly at the hilus.

The cervical lymph node biopsy showed a chronic granulomatous lymphadenopathy, with extensive central suppurative neutrophilic necrosis, suggestive of catscratch disease. The definitive diagnosis was obtained after a "Whartin Starry" stain that showed the presence of gram negative rod, indicative of Bartonella henselae (Figure 1).

Chlarythromycin was administered for five days in adjusted doses as an outpatient. The patient reported a progressive decrease in lymph node size and no other complaints after treatment.

Two weeks later, the patient was readmitted to the emergency room presenting an acute episode of left hemiplegia associated with no other symptoms. No seizures were reported and there was no mental status change. The spinal fluid profile was normal.

Corticosteroids were empirically administered following image procedures. A contrast-enhanced MRI showed an inconclusive hyperintense lesion located at the right frontoparietal transition at $\mathrm{T}_{2}$-weighted image with no contrast enhancement (Figures 2 and 3). No arteriovenous malformations, masses or aneurysms were observed on magnetic resonance angiography.
After five days as an inpatient, the boy was dismissed with partial recovery of neurological function and with no further medication. Complete recovery was observed 20 days later on follow-up examination.

\section{Discussion}

Cats are healthy carriers of $B$. henselae and can be bacteraemic for months to years. The organism can be transmitted from cat to cat by the cat flea, but not by direct contact between animals [4].

Although CSD is found all over the world, it is an uncommon disease. One estimative by the Centers for Disease Control found that there were 2.5 cases of CSD per 100,000 people per year in the United States. In one study using a United States national database, the incidence of CSD was approximately 9 to 10 cases per 100,000 people per year. The majority of individuals who contract CSD are immunocompetent and are under the age of 21 [5].

The most commonly involved lymph nodes in CSD are the anterior cervical, axillary, inguinal, femoral, preauricular, supraclavicular, and epitrochlear nodes [1]. The nodes tend to be large, ranging from 1 to $6 \mathrm{~cm}$ at the time of maximum size, but can be as large as 10-13 $\mathrm{cm}$. Most of the nodes are tender, and late in the course of disease the nodes often develop erythema of the overlying skin, and occasionally suppurate (10 to 15 percent). Approximately 85 percent of patients have solitary lymphadenopathy [1].

The usual course of the disease is one to four months. Some cases are more severe and more protracted, and may last up to six to seven months. Other reports have described persistence of enlarged nodes for one to three years [2].

The laboratory tests include two serologic methods for antibody detection: indirect fluorescence assay (IFA) and enzyme immunosorbent assay (EIA). Prior to the development of a confirmatory serologic test for CSD, intradermal skin testing was an option. However, with the development of specific laboratory tests it is no longer recommended [1]. 
Figure 1. ("Whartin Starry" stain): Presence of a Gram-negative rod (white circle), indicative of Bartonella henselae.

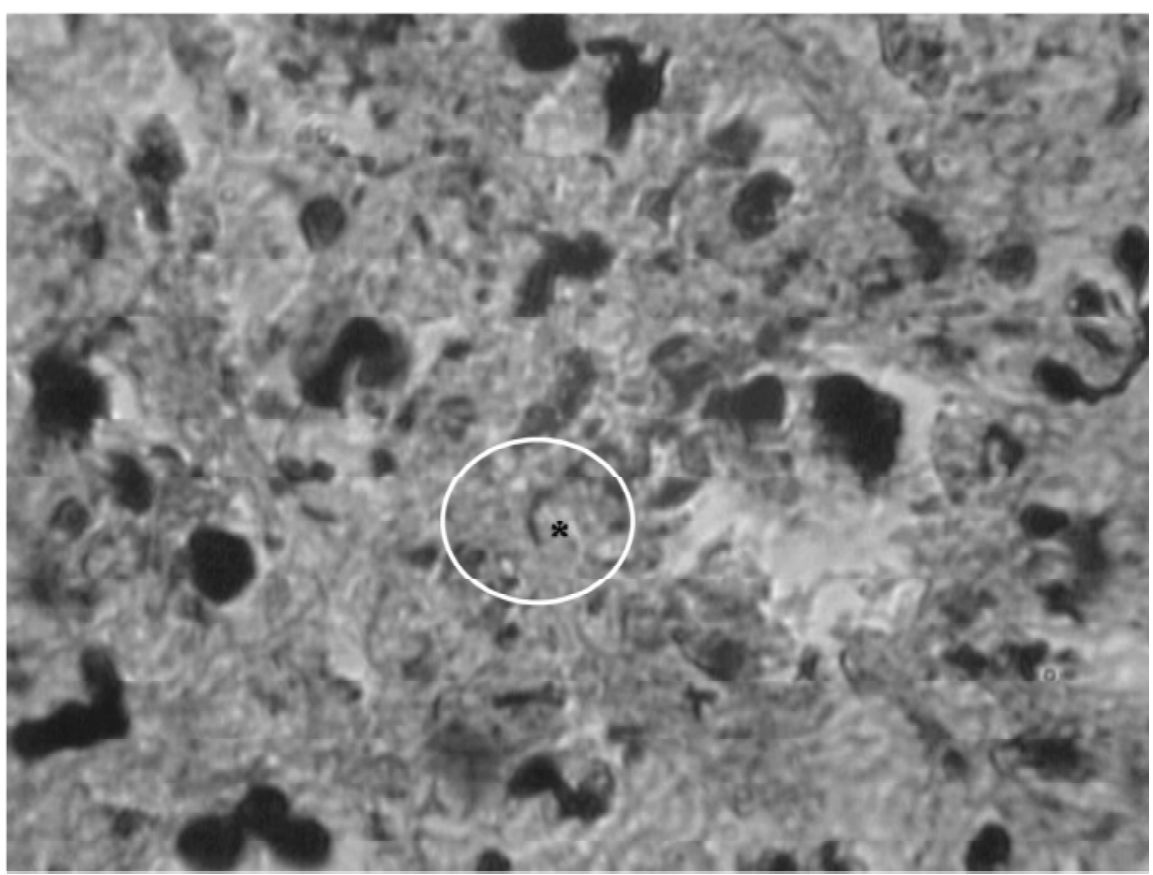

Figures 2 and 3. (Brain MRI): Hyperintense lesion located at the right frontoparietal transition at $T_{2}$-weighted image with no contrast enhancement (white circle).

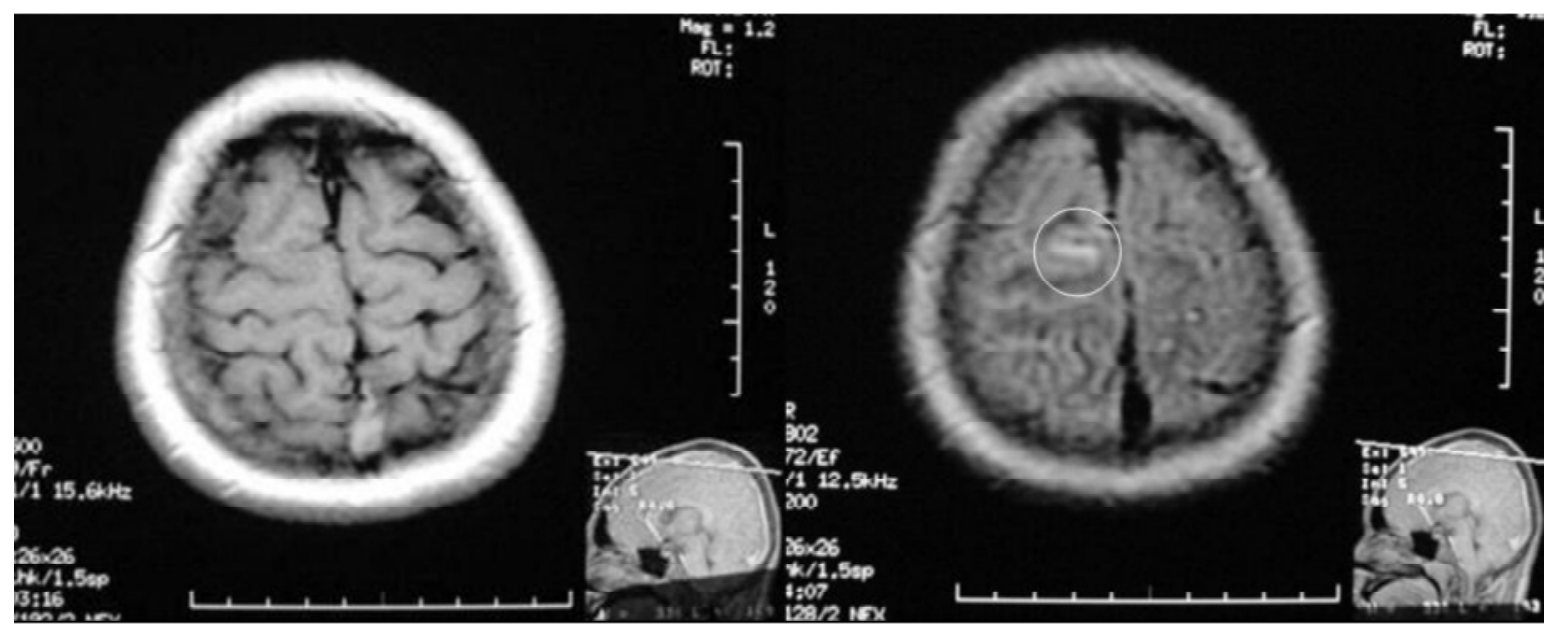


Isolation of $B$. henselae by culture can provide a definitive diagnosis, but bacterial isolation from tissue samples remains very difficult. A positive polymerase chain reaction (PCR) of a tissue specimen can also confirm the diagnosis $[4,5]$.

Histopathological examination of the primary lesions in the skin shows acellular areas of necrosis in the dermis, surrounded by multiple layers of histiocytes and epithelioid cells. Multinucleated giant cells may be present. Alymph node biopsy reveals findings similar to those of the skin samples, but they usually are nonspecific and depend upon the stage of the disease. Pleomorphic $B$. henselae bacilli in chains, clumps, or filaments can be seen with a Warthin-Starry silver impregnation stain $[4,5]$.

Cat scratch disease comes to complete resolution in most cases and surgical drainage is not suggested. Although the benefits of antimicrobial therapy are not completely proved, it is recommended to use Azithromycin $[1,4]$. There is no objective evidence of benefit using steroids [6,7].

Cat scratch disease is rarely associated with neurological complications. Nevertheless, among neurological complications associated with CSD, by far the most common (90\%) is encephalopathy, which occurs in $2 \%$ to $3 \%$ of patients, with onset varying from a few days to months after diagnosis of CSD. Typically, encephalopathy associated with CSD occurs within 2 to 3 weeks, presenting with headache and change in mental status. Convulsions are relatively common (46\%). In addition, other neurological manifestations that may accompany encephalopathy are neuroretinitis, Parinaud's oculoglandular syndrome, myelopathy, radiculopathy, facial nerve paresis, cerebral arteritis, and chronic inflammatory demyelinating polyneuropathy [6-9].

The pathogenesis of CNS complications has not been elucidated. However avasculitic origin was suggested by Selby and Walker after demonstrating a cerebral arteritis in a 7-year-old girl with CSD [9].

The spinal fluid is usually normal, although there are some reports of pleocytosis. EEGs are usually consistent with encephalopathy. CT and MRI are usually within normal range and there are few reports of focal changes. The prognosis is good and there are very few reports of long-term sequels and no reports of deaths [10].

\section{References}

1. Vincent J.M., Demers D.M., Bass J.W. Cat Scratch Disease: A Review. Hawaii Department of Health, 2000.

2. Arisoy E.M., Correa A.G., Wagner M.L., Kaplan S. Hepatosplenic cat-scratch disease in children: Selected clinical features and treatment. Clinical Infectious Diseases 1999;28;778-84.

3. Hulzebos C.V., Koetse H.A., Kimpen J.L.L., Wolfs T.F.W. Vertebral osteomyelitis associated with cat-scratch disease. Clinical Infectious Diseases 1999;28;1310-2.

4. Chomel B.B. Cat-scratch disease and bacillary angiomatosis. Rev Sci Tech 1996;15(3);1061-73.

5. Little S. Cat Scratch Disease. In: CFA Health Committee [online]. Available at: www.cfainc.org. Accessed November 10, 2002.

6. Marra C.M. Neurologic complications of Bartonella henselae infection. Current Opinion in Neurology 1995;8(3);164-9.

7. Miller P., Bell W.E. Cat-scratch disease with encephalopathy. Clinical Pediatrics 1980;19(3);233-4

8. Lewis D.W., Tucker S.H. Central nervous system involvement in cat scratch disease. Pediatrics 1986;77(5);714-21.

9. Selby G., Walker G.L. Cerebral arteritis in cat-scratch disease. Neurology 1979;29;1413-8.

10. Carithers H.A., Margileth A.M. Cat-scratch disease, acute encephalopathy and other neurologic manifestations. AJDC 1991; 145:98-101. 